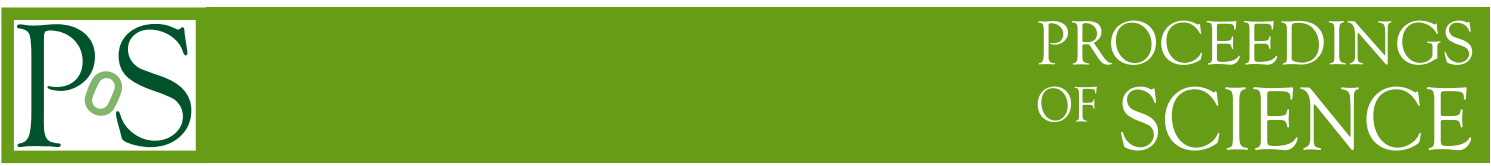

\title{
Performance of the ATLAS tau-lepton trigger at the LHC in Run 2
}

\author{
Eleni Myrto Asimakopoulou, on behalf of the ATLAS Collaboration \\ Uppsala University \\ E-mail: eleni.myrto.asimakopoulou@cern.ch
}

The ATLAS experiment has a rich physics program of Standard Model measurements and searches for physics Beyond the Standard Model involving tau leptons. Most of these analyses depend on an efficient tau-lepton trigger that can cope with the overwhelming background from multi-jet events produced in proton-proton collisions at the Large Hadron Collider. The ATLAS trigger system is composed of two stages. At Level-1, tau leptons are reconstructed as energy deposits in neighboring towers of calorimeter cells. The High Level Trigger (HLT) exploits the full calorimeter granularity as well as inner-detector tracks, and runs reconstruction and identification algorithms similar to those used in the offline reconstruction. The performance of the tau-lepton trigger in ATLAS Run-2 data will be discussed, and trigger efficiencies measured with a tag-andprobe method will be presented. An emphasis will be made on the improved HLT algorithms deployed in 2018 and mentioned below. The association of tracks to the energy deposit in the calorimeter was tightened to reduce the contamination from fake tracks at high pileup. An energy calibration based on a Boosted Regression Tree with improved energy resolution has replaced the simpler calibration based on pileup subtraction and a calorimeter response correction. An identification algorithm based on a Recurrent Neural Network was also deployed, which provides increased jet rejection compared to the previously-used Boosted Decision Tree identification algorithm.

XXIX International Symposium on Lepton Photon Interactions at High Energies - LeptonPhoton2019 August 5-10, 2019

Toronto, Canada 


\section{Introduction}

A large number of physics analyses carried out at the Large Hadron Collider (LHC) [1] are dependent on tau leptons. Tau leptons have a mass of $1777.86 \pm 0.12 \mathrm{MeV}$, a proper decay length of $87.03 \pm 0.15 \mu \mathrm{m}$ and decay hadronically with a $65 \%$ branching ratio (dominantly to pions). The ATLAS tau lepton trigger aims to reconstruct only the hadronic decay products, while the leptonic decays are identified by dedicated electron and muon triggers. The expected detector signature within ATLAS [2] is one or three tracks in the inner detector and a narrow isolated energy deposition in the calorimeters, with a significant background source being misidentified jets. The limitations on computing resources for offline storage and data processing impose the need of a trigger system for rate reduction. In ATLAS the data collection is carried out with a two-level trigger system [3]. During the full Run 2 proton-proton collision data taking the LHC delivered an integrated luminosity of $156 \mathrm{fb}^{-1}$, with ATLAS recording $139 \mathrm{fb}^{-1}$ for physics. The evaluation of the ATLAS tau-lepton trigger was performed with the full Run 2 data, while the newly added Recurrent Neural Network ID (discussed in Sec. 3) was evaluated with data collected between July and October of 2018 at $\sqrt{s}=13 \mathrm{TeV}$, corresponding to an integrated luminosity of $36.8 \mathrm{fb}^{-1}$.

\section{Tau Trigger}

The trigger decision is developed from the combination of a primary hardware based trigger, referred to as the Level-1 (L1) trigger, and a subsequent software based trigger, referred to as the High Level Trigger (HLT); achieving a rate decrease from $40 \mathrm{MHz}$ down to $100 \mathrm{kHz}$ (L1) and subsequently $1 \mathrm{kHz}$ (HLT). The main component of the L1 tau trigger is the L1Calo trigger which utilizes calorimeter (electromagnetic (EM) and hadronic (HAD)) information for the definition of a core and an isolation region for each tau candidate. The core region is defined as a square $0.2 \times 0.2$ region in $\Delta \eta \times \Delta \phi$ space, where the transverse energy $\left(E_{\mathrm{T}}\right)$ of the visible hadronic tau candidate ( $\tau_{\text {had-vis }}$ ) is defined as the $E_{\mathrm{T}}$ sum in the two most energetic neighboring central towers in the EM calorimeter and in the $\Delta \eta \times \Delta \phi=0.2 \times 0.2$ space in the HAD calorimeter. For each $\tau_{\text {had-vis }}$ candidate, an isolation cut-off is imposed in the annulus $0.2<\Delta R<0.4$ region $^{1}$ to reduce background. The cut-off is energy dependent and only affects $\tau_{\text {had-vis }}$ candidates up to $60 \mathrm{GeV}$ with an upper threshold of: $E_{\mathrm{T}}^{\mathrm{EM}_{\mathrm{isol}}[\mathrm{GeV}]} \leq\left(E_{\mathrm{T}}[\mathrm{GeV}] / 10+2 \mathrm{GeV}\right)$. The regions that satisfy these requirements provide Regions of Interest (RoIs) for the subsequent triggering steps. In the case of combined di-lepton triggers $^{2}$, further background rate reduction is achieved by utilizing the L1Topo for performing $\Delta R$ cuts on the candidates in order to remove overlapping RoIs. Once the RoIs have been defined, the HLT applies a sequential list of algorithms to further reduce the rates of considered $\tau_{\text {had-vis }}$ candidates. The algorithms are applied in increasing order of CPU intensity, optimizing the total processing time. First a candidate preselection is performed using the calorimeter information from the seeded RoIs. The calorimeter clusters of interest are calibrated with a local hadronic calibration and their vectorial sum is used to define a "jet seed". The second HLT step is called Fast Track Finding (FTF) and is looking for tracks in the inner detector by performing of a two-stage pattern recognition algorithm on the "jet-seed". The first stage consists of a scan in a narrow $\Delta R=0.1$

\footnotetext{
${ }^{1} \Delta R$ is defined as $\Delta R:=\sqrt{\Delta \eta^{2}+\Delta \phi^{2}}$.

2 where an additional electron, muon or tau lepton is required in the event.
} 
region around the $\tau_{\text {had-vis }}$ candidate, along the full beamline $(|\Delta z|<225 \mathrm{~mm})$, in search of a lead track. If a lead track is found, a second scan is performed in a larger $\Delta R=0.4$ cone and narrow $|\Delta z|<7 \mathrm{~mm}$ region around the lead track, in search of additional tracks. The selected candidates pass to the last step of the HLT where precision tracking algorithms are executed. The precise track information is used along with the candidate's calorimeter information for the calculation of a list of input variables to Machine Learning algorithms (Boosted Decision Trees (BDT), or Recurrent Neural Networks $(\mathrm{RNN})$ ) trained to produce a $\tau_{\text {had-vis }}$ identification score that is used for the final candidate selection. The track counting of the candidates is either performed at both the second FTF stage and the precision tracking stage (2017 trigger chains) or only at the the precision tracking stage (2018 trigger chains). The candidates are required to have one to three tracks or up to three tracks depending on whether the identification is performed with the BDT or the RNN algorithm.

\section{Tau Trigger Performance}

The evaluation of the trigger performance is carried out with the Tag and Probe method in data and Monte Carlo (MC) samples. The method requires the identification of a muon in the event (tag), using the lowest unprescaled trigger available, and the offline reconstruction of a $\tau_{\text {had-vis }}$ candidate (probe) (discussed in more detail in [4]). The targeted processes are $Z \rightarrow(\tau)(\tau) \rightarrow$ $\left(\mu v_{\mu} v_{\tau}\right)\left(\tau_{\text {had-vis }} \nu_{\tau}\right)$, due to its high statistics and good purity, and $t \bar{t} \rightarrow\left(b \mu v_{\mu}\right)\left(b \tau_{\text {had-vis }} \nu_{\tau} v\right)$, due to higher $\tau_{\text {had-vis }} p_{\text {T }}$ profile. The modeling is evaluated by comparing data with signal and background samples, generated in $\mathrm{MC}$, that have been reconstructed with the same algorithms as the data (Figure 1). The online candidate selection requires isolation and $E_{\mathrm{T}}>12 \mathrm{GeV}$ at $\mathrm{L} 1$ and $p_{\mathrm{T}}>$ $25 \mathrm{GeV}$ at the HLT, without any track requirements. It should be noted that only candidates matched within a $\Delta R<0.2$ of an offline $\tau_{\text {had-vis }}$ probe are considered for the modeling, causing a bias in the selection by the identification and track multiplicity requirements on the probe. The efficiency of the trigger selection is evaluated based on the fraction of offline $\tau_{\text {had-vis }}$ probes that successfully pass the tau trigger (Figure 2).
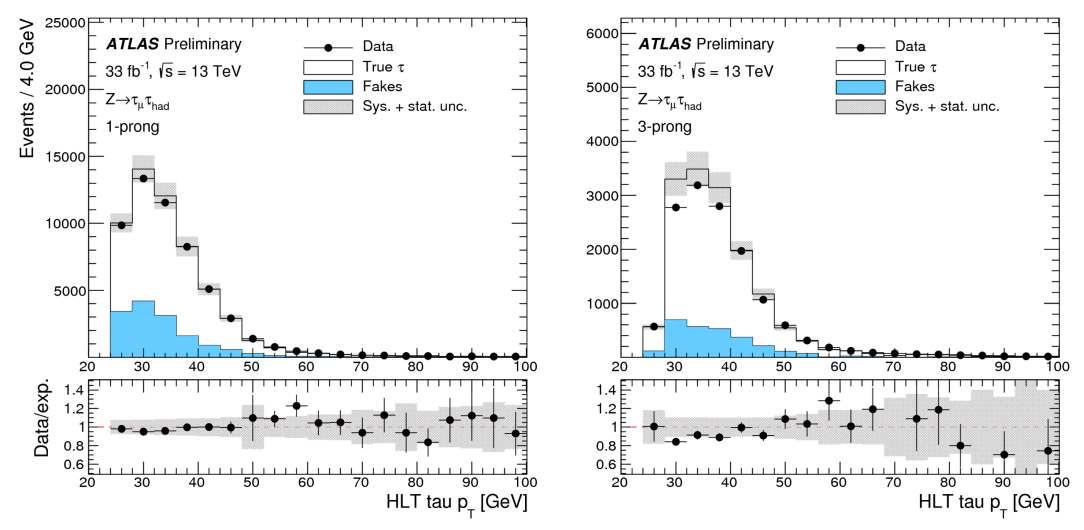

Figure 1: Distributions of online $\tau_{\text {had-vis }}$ candidates matched to an offline 1-prong (left) and 3-prong (right) $\tau_{\text {had-vis }}$ probe in the selected $Z \rightarrow \mu \tau_{\text {had-vis }} 3 v$ events. Figure from [4].

Three main improvements concerning track association, energy calibration and identification algorithms were included in the tau trigger during Run 2, increasing its performance. A list of new 

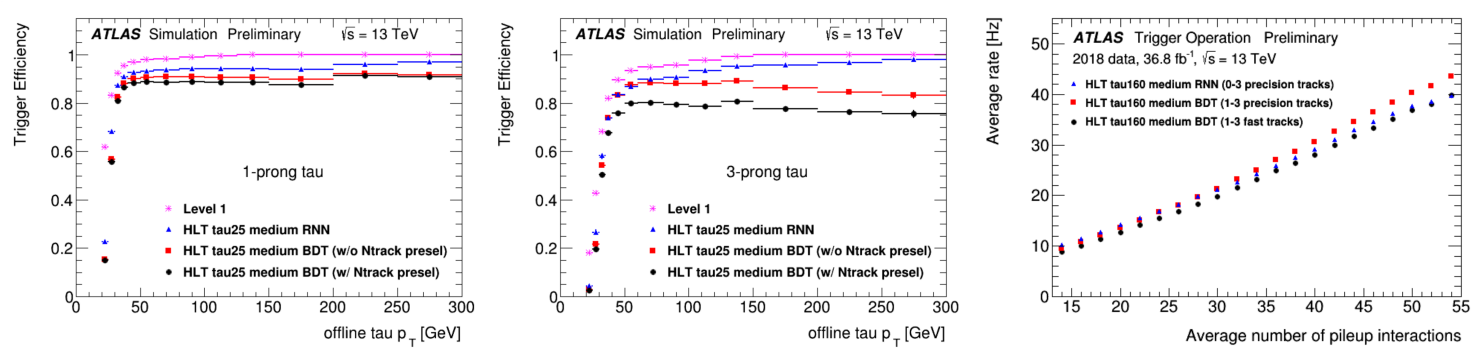

Figure 2: (Left, Center) Trigger efficiency for a candidate passing an $E_{\mathrm{T}}>12 \mathrm{GeV}$ threshold and a medium isolation requirement at $\mathrm{L} 1$, and an $E_{\mathrm{T}}>25 \mathrm{GeV}$ threshold at the HLT, as a function of the $p_{\mathrm{T}}$ of the matched 1-prong (left) and 3-prong (center) offline $\tau_{\text {had-vis }}$ probe. (Right) Average rate of single-tau triggers with an $E_{\mathrm{T}}$ threshold of $160 \mathrm{GeV}$ at the High Level Trigger (HLT) as a function of the average number of pileup interactions. The RNN trigger achieves rates comparable to, and even lower at high pileup, the BDT triggers, while using a looser track requirement. Figures from [5].

trigger chains were commissioned in 2018, introducing track counting only on precision tracks (ie. at the last stage of the HLT) and tighter $\Delta z_{0}$ requirements between the lead and additional tracks during selection. The changes were introduced to reduce fake track contamination and succeeded in recovering efficiency at high pileup, without causing significant rate changes. The energy calibration of the hadronic tau energy scale to the energy of the visible decay products during Run 2 was carried out with Boosted Regression Trees (BRT), instead of the pre-existing baseline calibration which utilized pileup subtraction and calorimeter response corrections. The BRT combines information from the baseline method along with calorimeter information, resulting in an improved energy resolution, especially at low $p_{T}$. Finally, a new identification algorithm, using a RNN [6], was introduced along with the nominal BDT algorithm. The RNN algorithm approaches tau identification as a sequence classification problem and takes as inputs the existing BDT input variables along with additional low-level track and cluster input variables, producing an identification score for the final trigger selection. The addition of the new algorithm increased jet rejection and allowed the recovery of misidentified one-track as zero-track, and three-track as two-track $\tau_{\text {had-vis }}$ candidates, making a significant impact on the trigger performance at low $p_{T}$ and high pile-up. The resulting trigger rate is comparable to that of the original BDT algorithm; an example is presented in Figure 2, where the RNN trigger achieves even lower rates at high pileup conditions, while applying looser track selection requirements.

\section{References}

[1] Evans, Lyndon and Bryant, Philip JINST 3 (2008) S08001.

[2] ATLAS Collaboration JINST 3 (2008) S08003.

[3] ATLAS Collaboration Eur. Phys. J. C 77 (2017) 317. 76 p.

[4] ATLAS Collaboration Tech. Rep. ATLAS-CONF-2017-061, https://cds.cern.ch/record/2274201.

[5] “ATLAS Tau Trigger Public Results."

https://twiki.cern.ch/twiki/bin/view/AtlasPublic/TauTriggerPublicResults.

[6] ATLAS Collaboration Tech. Rep. ATL-PHYS-PUB-2019-033, https://cds.cern.ch/record/2688062. 